\title{
Effect of numbers of turns of HPT on the development of exceptional ductility in pure magnesium
}

\author{
Roberto B. Figueiredo ${ }^{1 *}$, Pedro Henrique R. Pereira ${ }^{1}$, Terence G. Langdon ${ }^{2}$ \\ ${ }^{1}$ Department of Metallurgical and Materials Engineering, \\ Universidade Federal de Minas Gerais, Belo Horizonte, MG 31270-901, Brazil \\ ${ }^{2}$ Materials Research Group, Department of Mechanical Engineering, \\ University of Southampton, Southampton SO17 1BJ, UK
}

\begin{abstract}
The low ductility of magnesium at room temperature is usually attributed to an insufficient number of independent slip systems. Recent research has shown that refining the grain structure of pure magnesium promotes a breakdown in the Hall-Petch relationship at low strain rates and may lead to the development of exceptional ductilities. The report describes the evolution of microstructure and the mechanical behaviour of pure magnesium using different amounts of imposed plastic deformation by high pressure torsion (HPT). It is shown that the initial coarse grains undergo twinning followed by a gradual grain refinement. The flow stress at low strain rates decreases as the grain size is reduced, thereby confirming an inverse Hall-Petch behaviour. The elongation to failure increases with grain refinement and elongations over $300 \%$ are achieved after $1 / 2$ turn of HPT. These experimental data agree with a model for the low temperature deformation behaviour of fine-grained pure magnesium.
\end{abstract}

Keywords: ductility; Hall-Petch breakdown; high-pressure torsion; magnesium; ultrafine grains.

*corresponding author: figueiredo@demet.ufmg.br 


\section{Introduction}

Magnesium generally displays poor ductility at low temperatures and significant effort has been directed towards alleviating this problem. It is usually accepted that the limited number of independent slip systems prevent extensive plastic deformation ${ }^{[1]}$. Experiments on magnesium single crystals revealed easy glide in [11 $2 \overline{2} 0]$ basal slip and rapid hardening in non-basal slip at low temperatures ${ }^{[2]}$ and showed also that the $\{11 \overline{2} 2\}\langle\overline{11} 23\rangle$ slip system operates at all temperatures despite a much higher critical resolved shear stress ${ }^{[3]}$. Twinning also contributes to plastic deformation ${ }^{[1]}$ and is largely active in polycrystalline magnesium ${ }^{[4]}$.

It is now known that grain refinement affects the deformation mechanism of magnesium and its alloys. It was reported that grain refinement reduces the activation stress ratio between non-basal to basal slip due to grain boundary compatibility stresses in an AZ31 alloy ${ }^{[5]}$. However, experiments on pure magnesium showed an increase in the basal slip activity with decreasing grain size ${ }^{[6]}$. The overall contribution of twinning to the plastic deformation was found to decrease with grain refinement ${ }^{[7]}$. This agrees with a change in the shape of the stressstrain curves observed in compression testing where a coarse-grained sample of pure magnesium exhibited a sigmoidal shape, typical of twinning-assisted deformation, and a finegrained sample exhibited a near perfect plastic behaviour at low strain rates ${ }^{[8]}$.

Accordingly, grain refinement also affects the ductility. A fine-grained structure enables grain boundary sliding to occur at high temperatures and this deformation mechanism is responsible for superplasticity in which a material exhibits elongations over $400 \%$ in tension ${ }^{[9,10]}$. The development of severe plastic deformation techniques ${ }^{[11]}$ and their use to process magnesium and its alloys provided the opportunity to produce ultrafine-grained samples with grain sizes smaller than $1 \mu \mathrm{m}$. Such grain refinement may reduce the temperature lower bound for superplasticity and elongations over $400 \%$ have been reported at temperatures as low as 473 $\mathrm{K}$ in a commercial alloy ${ }^{[12]}$ and even at room temperature in a $\mathrm{Mg}-\mathrm{Li}$ alloy ${ }^{[13]}$. 
Exceptional elongations, within $\sim 40-400 \%$, were reported also in fine-grained pure magnesium and magnesium alloys at low and moderate temperatures. Although this range of elongation fails to characterise superplasticity, it is nevertheless remarkably high compared to coarse-grained magnesium. Thus, an early report documented unusual high elongations in pure magnesium with a grain size of $\sim 2 \mu \mathrm{m}{ }^{[14]}$. Recently, multiple research groups reported exceptional ductilities in pure magnesium with grain sizes smaller than $3 \mu \mathrm{m}^{[8,15-17]}$ including a maximum elongation of $\sim 360 \%$ in tension at room temperature ${ }^{[16]}$. Elongations over $200 \%$ were also reported in an AZ31 alloy tested in tension at $373 \mathrm{~K}^{[18]}$. The explanation for these exceptionally high ductilities has been attributed to the partial contribution of grain boundary sliding to the deformation and this proposal is supported by a concomitant increase in the strain rate sensitivity in these materials. It is known that severe plastic deformation by high-pressure torsion (HPT) refines the grain structure of pure magnesium to a range in which an increase in ductility is observed. Accordingly, the present research was undertaken in order to evaluate the effect of the number of turns in HPT on the development of exceptional ductilities in commercial purity (CP) magnesium.

\section{Experimental material and procedures}

The commercial purity (CP) magnesium used in this study was supplied by RIMA (Bocaiúva-Mg, Brazil) in the form of as-cast ingots displaying grains with an average size of $\sim 300 \mu \mathrm{m}$. The ingots were machined to round bars having dimeters of $\sim 10 \mathrm{~mm}$ and these bars were cut using a low-speed diamond saw to produce discs with thicknesses of $\sim 1.0 \mathrm{~mm}$. The surfaces of these CP-Mg samples were further ground using abrasive papers down to a thickness of $\sim 0.85 \mathrm{~mm}$.

The CP-Mg discs were processed by quasi-constrained HPT processing ${ }^{[19]}$ at room temperature $(\mathrm{RT} \approx 293 \mathrm{~K})$ using a nominal pressure of $6.0 \mathrm{GPa}$ and a rotation rate of $\sim 2 \mathrm{rpm}$. 
Samples were compressed within the HPT anvils $(\mathrm{N}=0)$ and processed up to $1 / 8,1 / 2,2$ and 10 turns.

Two tensile specimens were machined from each disc using electrical discharge machining. These specimens had a gauge length of $\sim 1.0 \mathrm{~mm}$ and a uniform gauge width of $\sim 0.9$ $\mathrm{mm}$, but displayed a somewhat convex surface due to the elastic distortion of the anvils during HPT processing. Accordingly, the tensile specimens were ground using grinding papers and finally polished using $0.06 \mu \mathrm{m}$ silica colloidal to obtain mirror-like flat surfaces having thicknesses of $\sim 0.6 \mathrm{~mm}$. Thereafter, these work-pieces were tested in tension using a Zwick Z030 testing machine operating at a constant rate of cross-head displacement. Tests were performed on the CP-Mg specimens processed to different numbers of HPT turns at RT using initial strain rates $(\dot{\varepsilon})$ of $10^{-5}, 10^{-3}$ and $10^{-1} \mathrm{~s}^{-1}$

The load and displacement data were recorded during tensile testing, converted into plots of true stress $(\sigma)$ vs true strain $(\varepsilon)$ assuming uniform deformation along the gauge area of the CP-Mg specimens. A Wild M420 stereoscope was used to obtain images of the tensile specimens immediately after testing and to measure the final lengths of the gauge sections of the samples.

Prior to tensile testing, the microstructures of the $\mathrm{CP}-\mathrm{Mg}$ tensile specimens were examined through optical microscopy (OM) using an Olympus BX51 optical microscope and scanning electron microscopy (SEM) using a JSM6500F thermal field emission SEM. The sample preparation involved grinding using abrasive papers followed by polishing using $1 \mu \mathrm{m}$ diamond paste and $0.06 \mu \mathrm{m}$ silica colloidal. The grain boundaries and other microstructural features such as twins were revealed after final polishing and the mean linear intercept lengths were estimated in the SEM images using the linear intercept method. 


\section{Experimental results}

Figure 1 displays the variation of true stress $(\sigma)$ as function of true strain $(\varepsilon)$ for $\mathrm{CP}-\mathrm{Mg}$ samples processed by HPT and subsequently pulled to failure at RT using strain rates ranging from $10^{-5}$ to $10^{-1} \mathrm{~s}^{-1}$. It is readily noted from these plots that the mechanical strength and ductility of the CP-Mg samples depend strongly on the amount of straining imposed during HPT processing. This material exhibits lower ductility after compression within the HPT anvils compared with the remaining processing conditions shown in Fig. 1. Furthermore, the compressed material displays higher flow stresses when pulled to failure at $10^{-5} \mathrm{~s}^{-1}$ and lower peak stresses for tests performed at $\dot{\varepsilon} \geq 10^{-3} \mathrm{~s}^{-1}$.

Inspection of Fig. 1 consistently reveals that increasing the number of HPT turns $(N)$ from $1 / 8$ to 2 leads to a significant increase in the maximum strains achieved in pure magnesium during tensile testing at RT. This is more prominent for the CP-Mg samples processed through $1 / 2$ and 2 revolutions of HPT and tested at $10^{-5} \mathrm{~s}^{-1}$ as these specimens underwent true strains higher than 1.3 without failure. By contrast, the material processed up to 10 turns of HPT exhibits higher flow stresses and fractures at lower strains by comparison to samples processed by $1 / 2$ and 2 turns. It should be further noted that there is a general trend for a reduction of the flow stresses of magnesium for tests conducted at decreasing strain rates.

Figure 2 shows the overall shapes of the CP-Mg specimens after tensile testing at RT using a strain rate of $10^{-5} \mathrm{~s}^{-1}$. The values of the elongation to failure $\left(\Delta \mathrm{L} / \mathrm{L}_{0}\right)$ are displayed at the righthand side of the deformed specimens for each testing condition. It follows from Fig. 2 that the CP-Mg samples processed up to 1/8 HPT turn exhibit elongations $\leq 60 \%$ and fracture at a plane approximately orthogonal to the tensile axis. On the other hand, $\Delta \mathrm{L} / \mathrm{L}_{0} \geq 320 \%$ for specimens initially processed by either $1 / 2$ or 2 turns of HPT and these samples develop a fairly diffuse neck during plastic straining. It is also apparent from Fig. 2 that the room temperature ductility 
of magnesium diminishes by increasing the number of HPT turns from 2 to 10 . Nonetheless,

the samples processed through 10 HPT turns continue to exhibit a distinct neck and achieve an elongation of $150 \%$ which is $\sim 2.5 \times$ higher than the value obtained for the samples processed by $1 / 8$ turn and tested in tension under an equivalent condition.

In order to compare the elongations to failure achieved at the various conditions of HPT processing used in this investigation, Fig. 3 shows plots of elongation vs strain rate for CP-Mg samples processed through different numbers of HPT turns and pulled to failure at RT using strain rates from $10^{-5}$ to $10^{-1} \mathrm{~s}^{-1}$. It is demonstrated in Fig. 3 that the room temperature ductility of magnesium tends to increase with increasing amounts of torsional straining imposed by HPT processing with the exception of the material processed up to 10 HPT turns.

For tests performed at RT using $\dot{\varepsilon}=10^{-1} \mathrm{~s}^{-1}$, the Mg specimens exhibit elongations which are below $50 \%$ regardless of the numbers of turns used during processing by HPT. However, the values of the elongations to failure consistently increase with decreasing strain rate. It is also evident in these plots that the room temperature ductility of magnesium at RT is further enhanced through HPT in samples processed up to either $1 / 2$ or 2 turns as $\Delta \mathrm{L} / \mathrm{L}_{0}>140 \%$ for tensile tests carried out at $\dot{\varepsilon}=10^{-3} \mathrm{~s}^{-1}$.

Figure 4 displays plots of the true stress at $\varepsilon=0.05$ as a function of the testing strain rate for CP-Mg discs originally processed by HPT using a rotation rate of $\sim 2 \mathrm{rpm}$ and further tested in tension at RT at various strain rates. The slopes of these curves correspond to the strain rate sensitivity $\left(m=[\partial \log \sigma / \partial \log \dot{\varepsilon}]_{T}\right)$ and it is readily apparent that the $m$ values of the HPTprocessed material increase with decreasing strain rate. For strain rates within the interval of $10^{-5}-10^{-3} \mathrm{~s}^{-1}$, the CP-Mg specimens subjected to any amount of torsional straining exhibit a strain rate sensitivity at RT of $\sim 0.2$, whereas $m<0$ for the material solely compressed within the quasi-constrained anvils. This negative value of $m$ at $\varepsilon=0.05$ may be associated with the 
occurrence of tension twinning during RT deformation as reported elsewhere for the AZ31 Mg

alloy tested in tension under comparable conditions ${ }^{[20]}$.

An examination of Figs 3 and 4 reveals that the CP-Mg specimens exhibiting exceptionally high ductility ( $\left.\Delta \mathrm{L} / \mathrm{L}_{0} \geq 320 \%\right)$ correspond to the experimental datum points showing the lowest flow stresses in Fig. 4 and they lie within the strain rate region in which $m \approx 0.2$. This indicates that grain boundary sliding (GBS) probably contributes to a significant amount of the plastic straining in the material processed to $1 / 2$ and 2 turns of HPT during deformation at $\dot{\varepsilon} \leq 10^{-3} \mathrm{~s}^{-1}$.

The overall appearance of the microstructures in the gauge lengths of the specimens prior to tensile testing is shown in Fig. 5 for all conditions. It is readily apparent that the material subjected to compression without any rotation in HPT displays coarse grains with a size comparable to the gauge width. These coarse grains contain many twins which are due to the compression deformation. The reduced number of grains along the gauge length in this case is expected to introduce a significant texture effect in the mechanical response of the material. The material processed to $1 / 8$ turn displays the remainder of the original coarse-grained structure but it is clearly deformed. The $\mathrm{CP}-\mathrm{Mg}$ specimens processed to $1 / 2,2$ and 10 turns exhibit homogeneous structures and the original coarse grains are not visible which confirms that grain refinement took place throughout the gauge length.

Figure 6 shows higher magnification details of the microstructures of the material processed to different numbers of turns of HPT. Significant twinning activity is observed in the material subjected to compression $(\mathrm{N}=0)$ but there is no evidence for any grain refinement. The material processed to $1 / 8$ turn displays a heterogeneous structure in which coarse grains, with evidence of twinning, are surrounded by refined grains. The samples processed to $1 / 2$ turn and to 2 turns exhibit a uniform structure with equiaxed ultrafine grains. The similarity of the grain structure in these samples suggests a saturation in grain refinement but the grain structure changes significantly after 10 turns. Thus, the grain size increases and the aspect ratio increases 
considerably. The average linear intercept lengths were $\sim 3.3 \mu \mathrm{m}, \sim 0.63 \mu \mathrm{m}, \sim 0.60 \mu \mathrm{m}$ and $\sim 1.2$

$\mu \mathrm{m}$ for the material processed to $1 / 8,1 / 2,2$ and 10 turns, respectively.

\section{Discussion}

It is known that HPT processing of magnesium and magnesium alloys promotes grain refinement and the mechanism of grain refinement differs from other metallic materials ${ }^{[21,22]}$. A multi-modal distribution of grain sizes is observed in the early stage of deformation due to the formation of ultrafine grains along the grain boundaries of the initial coarse grains. After a sufficient amount of straining is able to refine the core of the original coarse grains, a homogeneous distribution of ultrafine grains is achieved. The present results confirm this mechanism of grain refinement. The initially coarse grains undergo significant twinning during the compression stage of HPT, a bimodal distribution of grain sizes is observed after 1/8 turn due to partial refinement of the original structure and a homogeneous distribution of ultrafine grains is observed after 1/2 turn. This is in agreement with EBSD characterisation of a similar material processed to $1 / 8$ and to $1 / 2$ turn of HPT using similar processing parameters except for a slower rotation rate of $1 \mathrm{rpm}{ }^{[16]}$. However, the grain structure of the material processed to 10 turns displays considerable grain growth and differs from the microstructure observed in a similar material processed to the same number of turns. This discrepancy is attributed to the different rotation rate during HPT processing which is two times faster in the present experiments. It has been shown that the deformation heating during HPT is directly proportional to the rotation rate ${ }^{[23,24]}$ and therefore the heating in the present experiments is expected to be 2 times higher. Also, the heating of the material is lower in the early stages of processing which explains the finer grain sizes in the samples processed to 1/8,1/2 and 2 turns.

The initial flow stress observed at the lowest strain rate $\left(10^{-5} \mathrm{~s}^{-1}\right)$ shows clearly an inverse Hall-Petch behaviour where the material with the coarser structure $(N=0)$ displays the highest stress and the material with the finer structure $(N=1 / 2$ and $N=2)$ the lowest. A breakdown in 
the Hall-Petch relationship for fine-grained pure magnesium at low strain rates was reported

earlier and the critical grain size was estimated as several microns ${ }^{[17]}$. This agrees with the present results. Such a breakdown in the Hall-Petch relationship has been attributed to the increased role of grain boundary sliding as a deformation mechanism ${ }^{[16,17]}$.

Experimental evidence for the contribution of grain boundary sliding to the plastic deformation of fine-grained pure magnesium at room temperature includes observations of the polished surfaces of tensile specimens after testing and a significant increase in the measured strain rate sensitivity ${ }^{[16,17]}$. Observations of the offsets of markers on the polished surface revealed a contribution of GBS of over $10 \%$ of the total strain ${ }^{[17]}$. It is worth noting that an increase in the strain rate sensitivity was reported in magnesium processed to $1 / 2$ turn and to further turns of HPT ${ }^{[16]}$. This agrees with the range of numbers of turns for which an increase in elongation was observed in the present experiments.

Despite the increased activity of GBS in fine-grained pure magnesium at room temperature, the experimental results are not in agreement with the theoretical prediction for GBS-controlled deformation. This deformation mechanism is associated with a stress exponent of 2 , an inverse grain size exponent of 2 and grain boundary diffusion in the rate controlling equation ${ }^{[25]}$. A detailed analysis of the data for fine-grained pure magnesium revealed a stress exponent of 5, an inverse grain size exponent of 2.5 and a reduced activation energy for diffusion of $\sim 75 \mathrm{~kJ}$ $\mathrm{mol}^{-1}[16]$.

In order to evaluate the effectiveness of these parameters, the strain rates in the current experiments were normalised by the effect of temperature and grain size by considering $75 \mathrm{~kJ}$ $\mathrm{mol}^{-1}$ for the activation energy for diffusion and an inverse grain size exponent of 2.5 , and these data were then plotted as a function of the flow stress normalized by the shear modulus in Fig. 7. Data from other reports ${ }^{[8,15-17]}$ including using different mechanical testing, different grain sizes and different testing temperature, are also presented in Fig, 7 for comparison. It is worth 
noting that the grain size, $d$, in the present experiments was determined considering a relationship of $d=1.74 \times L$, where $L$ is the average linear intercept length. The majority of the reports in the literature use EBSD data analysis to determine the grain size which may introduce discrepancies when comparing the different results. Despite this problem, it is readily apparent that the data from these multiple reports agree well and follow a trend compatible with a stress exponent of 5 .

It is important to note that exceptional ductility was reported in all the reports from which data were included in Fig. 7. These exceptional ductilities include deformation in compression without failure up to $\sim 40 \%{ }^{[8]}$ and $\sim 80 \%{ }^{[15]}$ and elongations in tension up to $\sim 230 \%{ }^{[17]}$ and $\sim 360 \%{ }^{[16]}$. The present results confirm this trend of exceptional ductility and elongations over $300 \%$ were observed in the material processed to $1 / 2$ turn and to 2 turns and tested in tension at $10^{-5} \mathrm{~s}^{-1}$. This confirms that, although GBS is not the rate-controlling mechanism in these experiments, a contribution from sliding provides exceptional ductility for pure magnesium at room temperature.

\section{Summary and conclusions}

1- The compression stage of HPT introduces significant twinning activity in commercial purity coarse-grained magnesium. The deformation imposed by $1 / 8$ turn partially refines the microstructure and complete refinement is achieved after $1 / 2$ turn using a rotation speed of $\sim 2$ rpm.

2- The gradual refinement of the microstructure is associated with a concomitant change in the flow properties. An inverse Hall-Petch behaviour is observed at low strain rates and the maximum elongation increases with decreasing grain size.

3- An elongation of over $300 \%$ wa achieved after only 1/2 turn of HPT and this exceptional ductility was maintained in the material processed through up to 2 turns. 
4- The mechanical behaviour agrees with multiple reports in the literature and with an analysis suggesting a stress exponent of 5, an inverse grain size exponent of 2.5 and an activation energy of $\sim 75 \mathrm{~kJ} \mathrm{~mol}^{-1}$ for the rate-controlling mechanism in fine-grained pure magnesium at low temperatures.

\section{Acknowledgements}

RBF acknowledges financial support from CNPq (grant \#400407/2016-7), FAPEMIG (grant \#APQ-00580-15) and Serrapilheira Institute (grant \#Serra-1709-17750). PHRP acknowledges support from CNPq (grant \#443736/2018-9). 


\section{References}

[1] P. G. Partridge, Metallurgical Reviews, 1967, 12, 169.

[2] H. Yoshinaga, R. Horiuchi, Transactions of the Japan Institute of Metals, 1964, 5, 14.

[3] T. Obara, H. Yoshinga, S. Morozumi, Acta Metallurgica, 1973, 21, 845.

[4] N. Dixit, K. Y. Xie, K. J. Hemker, K. T. Ramesh, Acta Materialia, 2015, 87, 56.

[5] J. Koike, T. Kobayashi, T. Mukai, H. Watanabe, M. Suzuki, K. Maruyama, K. Higashi, Acta Materialia, 2003, 51, 2055.

[6] C. M. Cepeda-Jiménez, J. M. Molina-Aldareguia, M. T. Pérez-Prado, Acta Materialia, 2015, 84, 443.

[7] C. M. Cepeda-Jimenez, J. M. Molina-Aldareguia, M. T. Perez-Prado, Acta Materialia, 2015, 88, 232.

[8] R. B. Figueiredo, F. S. J. Poggiali, C. L. P. Silva, P. R. Cetlin, T. G. Langdon, Journal of Materials Science, 2016, 51, 3013.

[9] M. Kawasaki, R. B. Figueiredo, T. G. Langdon, Advanced Engineering Materials, 2016, $18,127$.

[10] T. G. Langdon, Journal of Materials Science, 2009, 44, 5998.

[11] R. Z. Valiev, R. K. Islamgaliev, I. V. Alexandrov, Progress in Materials Science, 2000, 45, 103.

[12] R. B. Figueiredo, T. G. Langdon, Scripta Materialia, 2009, 61, 84.

[13] K. Edalati, T. Masuda, M. Arita, M. Furui, X. Sauvage, Z. Horita, R. Z. Valiev, Scientific Reports, 2017, 7.

[14] J. A. Chapman, D. U. Wilson, Journal of the Institute of Metals, 1962-63, 91, 39.

[15] Z. Zeng, J.-F. Nie, S.-W. Xu, C. H. J. Davies, N. Birbilis, Nature Communications, 2017, 8, 972 .

[16] R. B. Figueiredo, S. Sabbaghianrad, A. Giwa, J. R. Greer, T. G. Langdon, Acta Materialia, 2017, 122, 322.

[17] H. Somekawa, T. Mukai, Metallurgical and Materials Transactions A, 2015, 46, 894.

[18] R. B. Figueiredo, P. H. R. Pereira, T. G. Langdon, Defect and Diffusion Forum, 2018, 385, 59.

[19] R. B. Figueiredo, P. R. Cetlin, T. G. Langdon, Materials Science and Engineering A, 2011, 528, 8198.

[20] Y. B. Chun, C. H. J. Davies, Materials Science and Engineering: A, 2011, 528, 5713.

[21] R. B. Figueiredo, T. G. Langdon, Advanced Engineering Materials, 2019, 21, 1801039.

[22] C. L. P. Silva, I. C. Tristão, S. Sabbaghianrad, S. A. Torbati-Sarraf, R. B. Figueiredo, T. G. Langdon, Materials Research, 2017, $20,2$.

[23] P. H. R. Pereira, R. B. Figueiredo, Y. Huang, P. R. Cetlin, T. G. Langdon, Materials Science and Engineering A, 2014, 593, 185.

[24] R. B. Figueiredo, P. H. R. Pereira, M. T. P. Aguilar, P. R. Cetlin, T. G. Langdon, Acta Materialia, 2012, 60, 3190.

[25] T. G. Langdon, Acta Metallurgica et Materialia, 1994, 42, 2437. 
Figures:
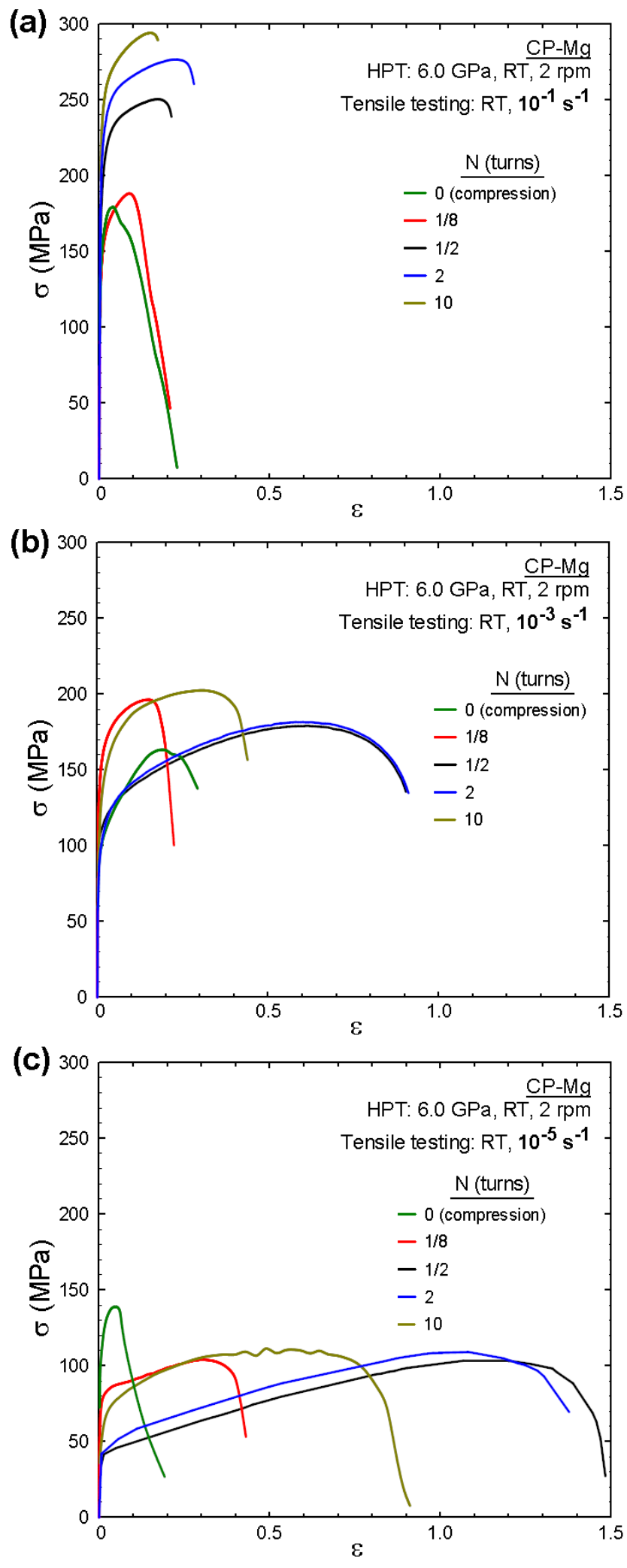

Fig. 1. True stress vs true strain curves for CP-Mg samples processed by HPT and then tested in tension at RT using strain rates of (a) $10^{-1}$, (b) $10^{-3}$ and (c) $10^{-5} \mathrm{~s}^{-1}$. 
CP-Mg

HPT: $6.0 \mathrm{GPa}, \mathrm{RT}, 2 \mathrm{rpm}$

Tensile testing: $\mathrm{RT}, 10^{-5} \mathrm{~s}^{-1}$

$\underline{\mathrm{N} \text { (turns) }}$

Elongation (\%)

0 (compression)

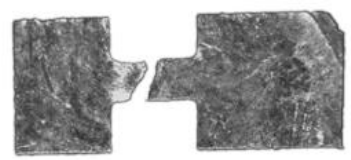

30

$1 / 8$

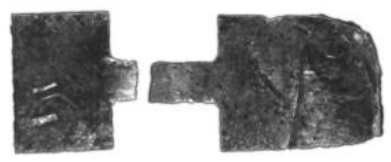

60

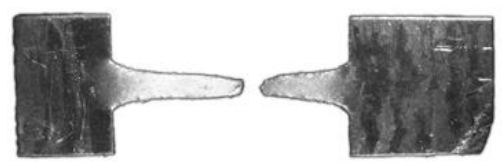

340

$1 / 2$

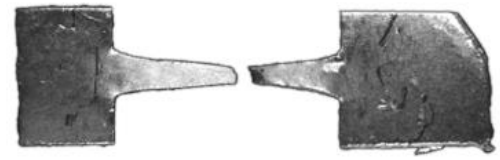

320

10

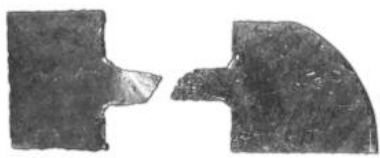

150

Fig. 2. Appearance of the deformed tensile specimens of CP-Mg samples processed by HPT and then pulled to failure at RT using an initial strain rate of $10^{-5} \mathrm{~s}^{-1}$. 


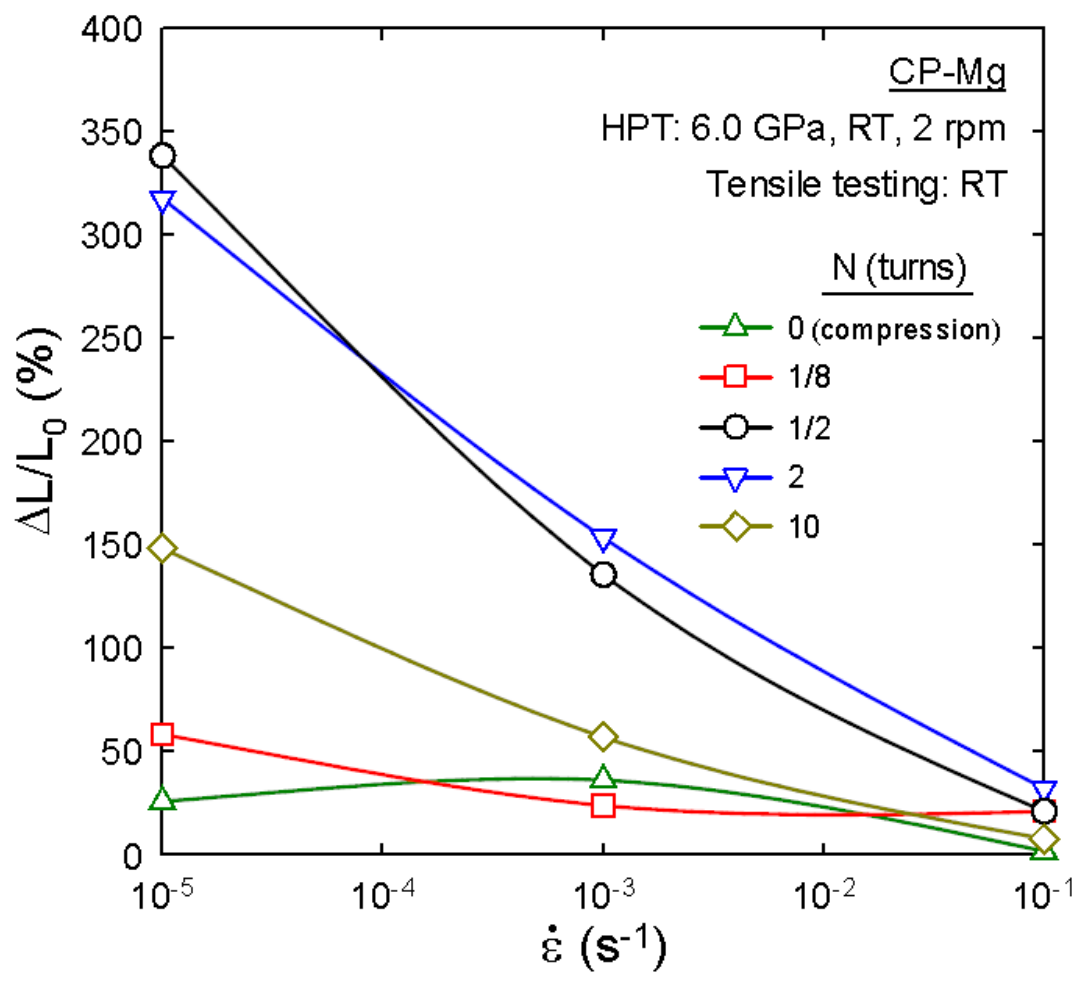

Fig. 3. Elongation vs strain rate for CP-Mg samples processed by HPT and then pulled to failure at RT using strain rates ranging from $10^{-5}$ to $10^{-1} \mathrm{~s}^{-1}$. 


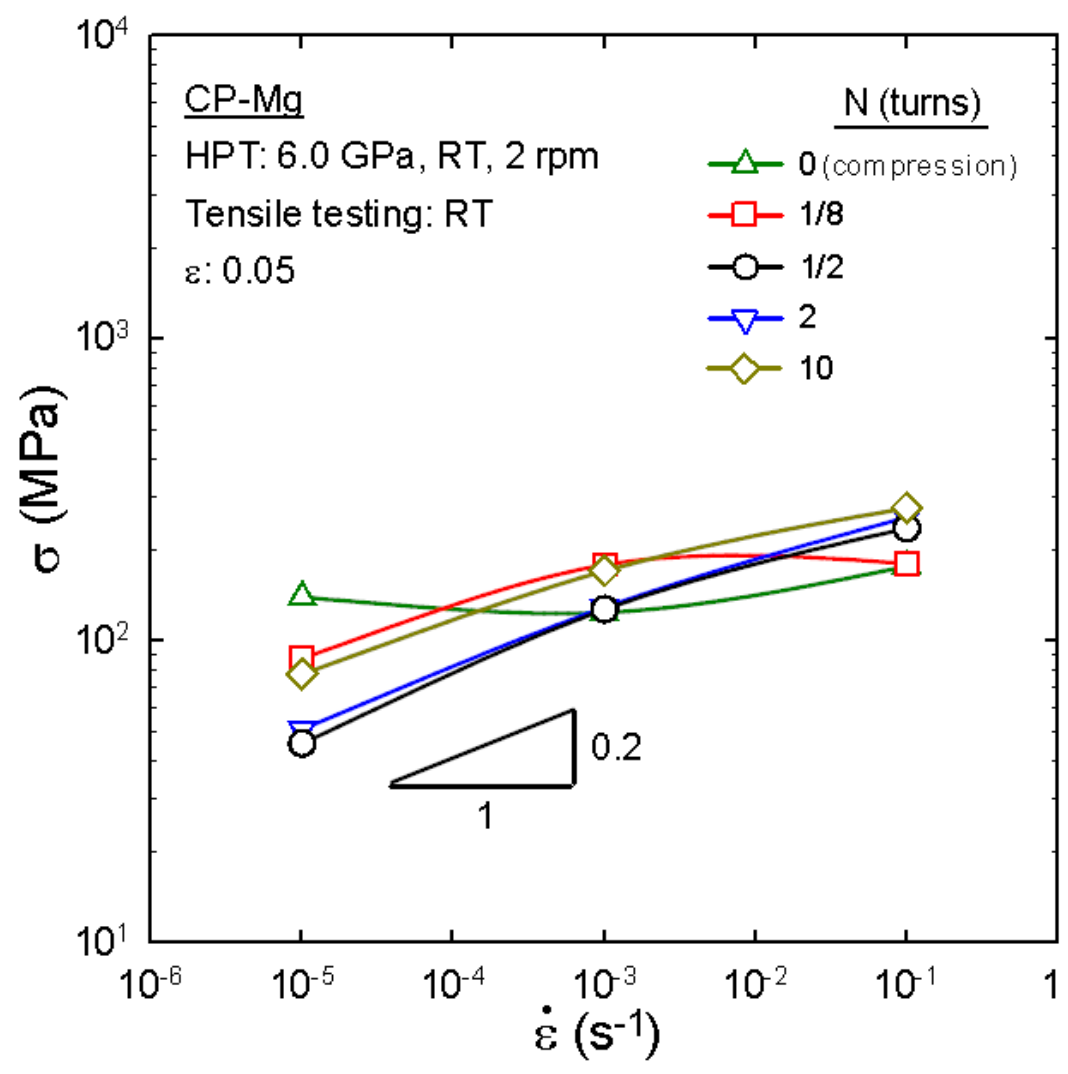

Fig. 4. True stress at $\varepsilon=0.05$ as a function of strain rate for $\mathrm{CP}-\mathrm{Mg}$ samples processed by HPT and pulled to failure at RT using strain rates ranging from $10^{-5}$ to $10^{-1} \mathrm{~s}^{-1}$. 


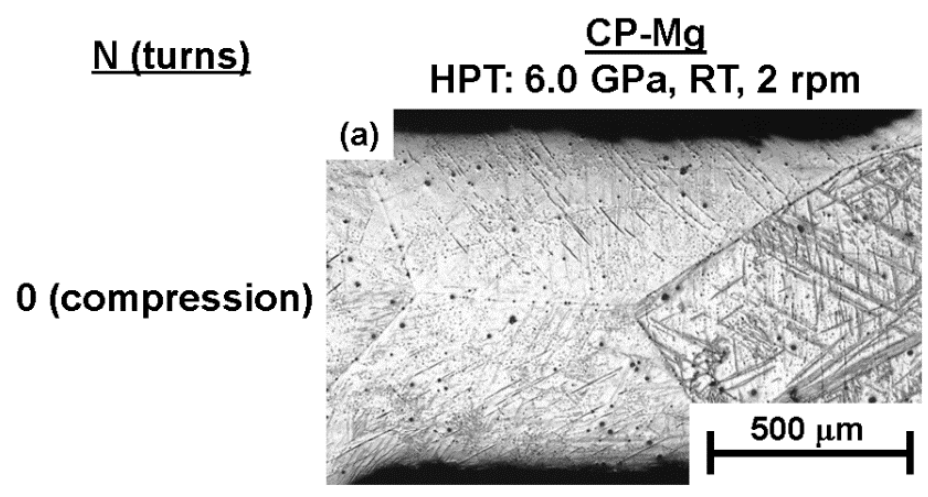

(b)

$1 / 8$

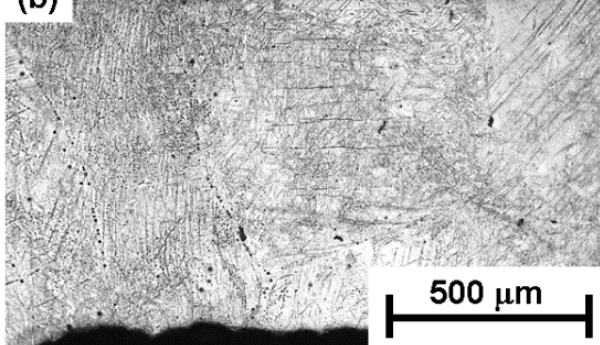

$1 / 2$

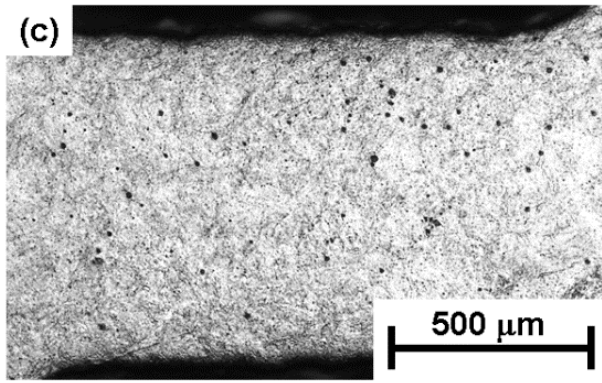

(d)

2

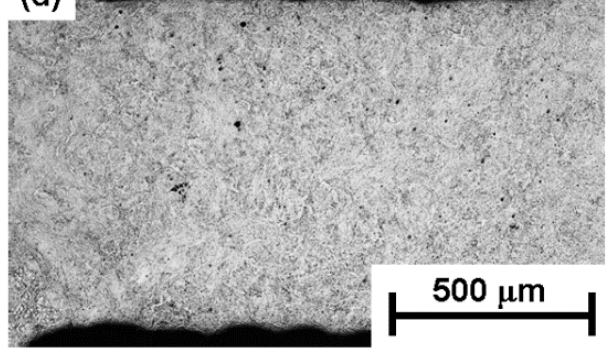

(e)

10

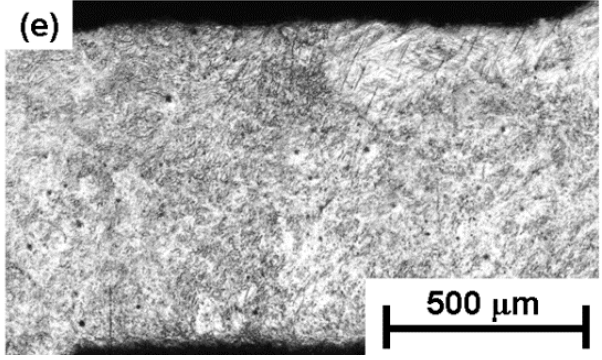

Fig. 5. OM images displaying the gauge sections of tensile specimens of CP-Mg samples (a) compressed within the HPT anvils and processed through (b) 1/8, (c) 1/2, (d) 2 and (g,h) 10 turns of HPT. 

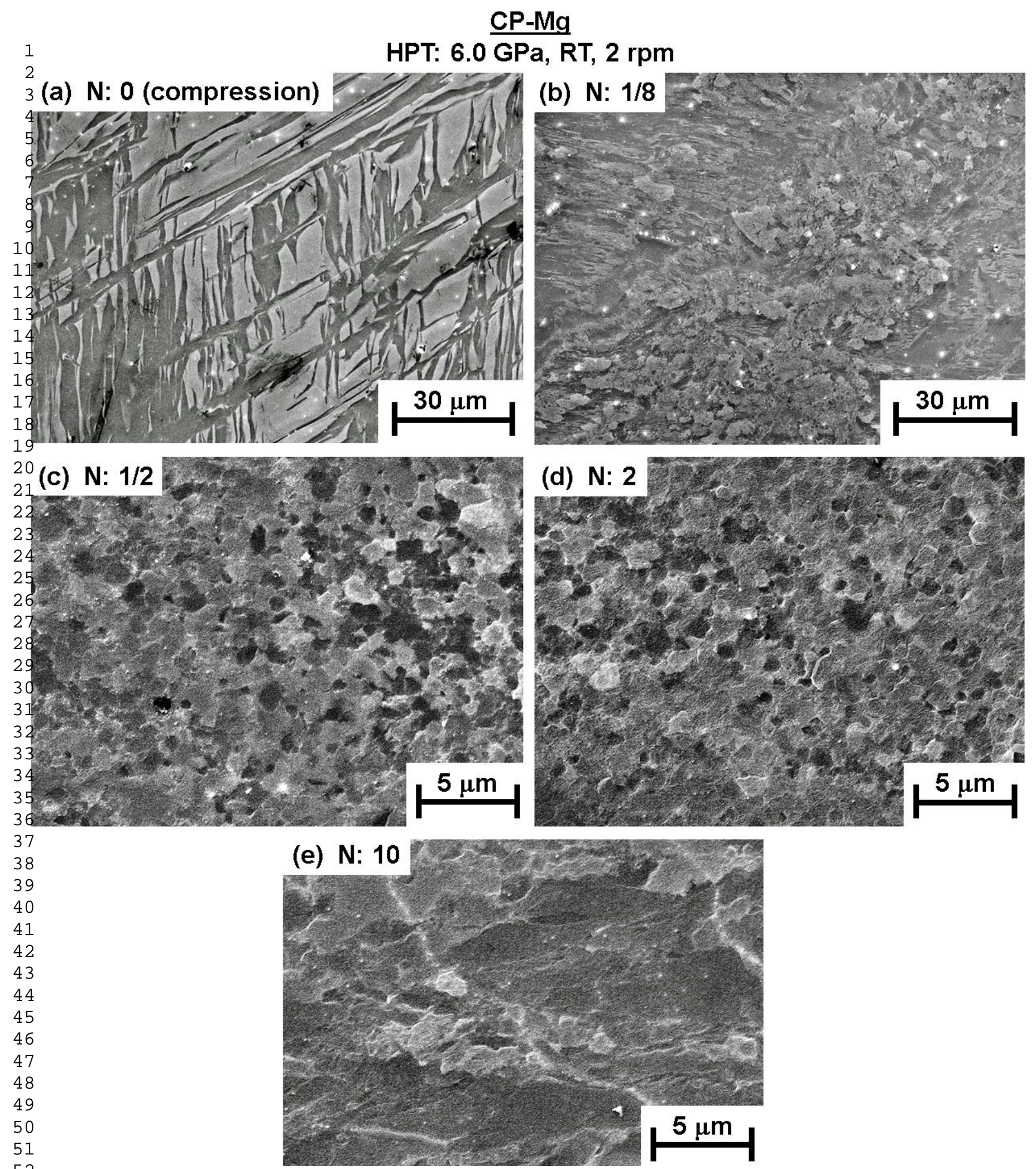

Fig. 6. SEM images displaying the gauge sections of tensile specimens of

CP-Mg samples (a) compressed within the HPT anvils and processed through (b) 1/8, (c) 1/2, (d) 2 and (e) 10 turns of HPT. 
1

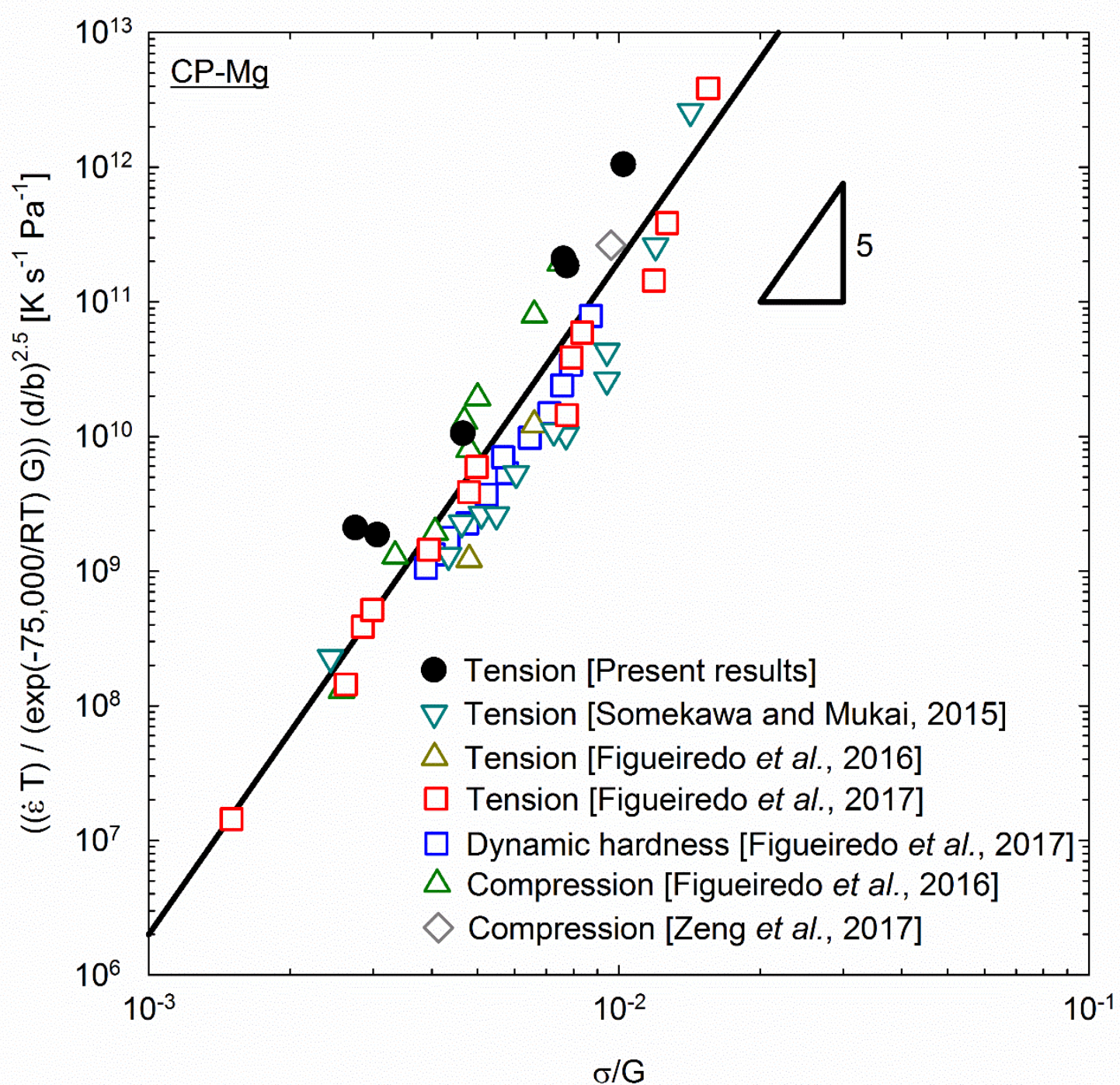

Fig. 7. Strain rate normalised by the grain size and temperature and plotted as a function of the flow stress normalised by the shear modulus ${ }^{[8,15-17]}$. 